\title{
INSTRUMENTATION AND METHODS FOR POWDER DIFFRACTION WITH SYNCHROTRON RADIATION
}

\author{
T. WROBLEWSKI \\ Hamburger Synchrotronstrahlungslabor HASYLAB \\ Deutsches Elektronen-Synchrotron DESY \\ Notkestr. 85, D-2000 Hamburg 52, Germany
}

\begin{abstract}
Powder diffraction covers a variety of applications ranging from high resolution structure determination to time-resolved experiments. This wide range can be covered if the properties of synchrotron radiation, especially the continuous spectrum and the high collimation, are properly taken advantage of. The latter property favours parallel beam geometry. The instrumental function for such a setup is aberration-free and can be derived using only the angular characteristics of its optical components, namely the divergence of the incoming beam and the reflection or transmission curves of the optical elements. The continuous spectrum allows either anomalous dispersion experiments or the use of a broad energy band for experiments on a short time scale. To achieve high flexibility in combination with the use of specialised setups for the different types of experiments a special diffractometer has been built at HASYLAB.
\end{abstract}

PACS numbers: 07.85. $+\mathrm{n}, 61.10 . \mathrm{Lx}$

\section{Introduction}

One requirement in the design of a powder diffraction experiment is high resolution because the measured peak positions only give the length of the corresponding reciprocal lattice vectors and not their orientation with respect to each other as single crystal diffraction does. If the crystal structure is close to a higher symmetry (for example if it is orthorhombic but not far from tetragonal) the powder pattern will exhibit strongly overlapping peaks. On the other hand, just these pseudosymmetrical substances tend to form twins so that single crystals are difficult to obtain. The next chapter will show how synchrotron radiation can provide intrinsically high resolution and how different optical elements affect the resolution function.

In many investigations it is not the ideal (perfect crystal) structure of the sample that is of interest but its real structure including strain, particle size, 
texture etc. To separate the influence of the sample from instrumental effects on the peak shape the instrumental profile function has to be known. Various approaches will be presented in Sec. 3 .

An important aspect in this discussion is that the instrumental function does not depend on the exact position and geometry of the sample. This property makes experiments like the investigation of moving objects (Sec. 4) and diffraction under grazing incidence (Sec. 5) possible.

Another application of powder diffraction is the investigation of structural changes. These experiments require special sample environments like furnaces and cryostats. To achieve high flexibility for different types of experiments a special diffractometer has been built at HASYLAB. This translation diffractometer and its special attachments will be described in Sec. 6. The investigation of structural changes that occur on a short time scale requires high intensity and a fast detection technique. The instrumentation for time-resolved experiments on the millisecond scale together with applications is described in Sec. 7.

The above-mentioned experiments make use of the high collimation and intensity of synchrotron radiation. The possibility of choosing an arbitrary wavelength from the white spectrum of synchrotron radiation permits experiments using anomalous dispersion effects, and these experiments will be described in Sec. 8 .

\section{Optics for powder diffraction with synchrotron radiation}

Synchrotron radiation exhibits an intense continuous spectrum and high collimation. Powder diffraction experiments are therefore mainly performed in parallel beam geometry. A monochromator selects a wavelength band from the continuous spectrum. The angle of diffraction by the sample is analysed by a receiving slit, a position-sensitive detector, a Soller collimator, or an analyser crystal. The resolution functions for these arrangements are given by a convolution of the angular and spectral distribution of the incoming beam with the transmission functions of all subsequent optical elements (including the sample) and the acceptance of the detector. Resolution functions for different arrangements are derived in [1]. Highest resolution is obtained by a triple-axis arrangement consisting of monochromator, sample, and analyser crystal. In the Gaussian approximation this resolution function is given by

$$
\begin{aligned}
& \text { FWHM }^{2}=\Delta_{3}^{2}+\left[\left(\tan \Theta_{3}-2 \tan \Theta_{2}\right) / \tan \Theta_{1}\right]^{2} \Delta_{1}^{2} \\
& +\left[\left(2 \tan \Theta_{2}-\tan \Theta_{1}-\tan \Theta_{3}\right) / \tan \Theta_{1}\right]^{2} \psi^{2},
\end{aligned}
$$

where $\theta_{1,2,3}$ are the Bragg angles of monochromator sample and analyser, $\Delta_{1,3}$ are the reflection widths of monochromator and analyser, and $\psi$ is the divergence of the incoming beam.

If the monochromator is not a perfect crystal or a $(+,-)$ double crystal arrangement but a single mosaic crystal the term depending on $\Delta_{1}$ must be replaced by

$$
\left.\left[2 \tan \Theta_{2}-2 \tan \Theta_{1}-\tan \Theta_{3}\right) / \tan \Theta_{1}\right]^{2} \Delta_{1}^{2} .
$$

Figure 2a shows the resolution function for the arrangement in Fig. 1 with Si 111 crystals as monochromator and analyser. From Eq. (1.1) it is clear that highest 


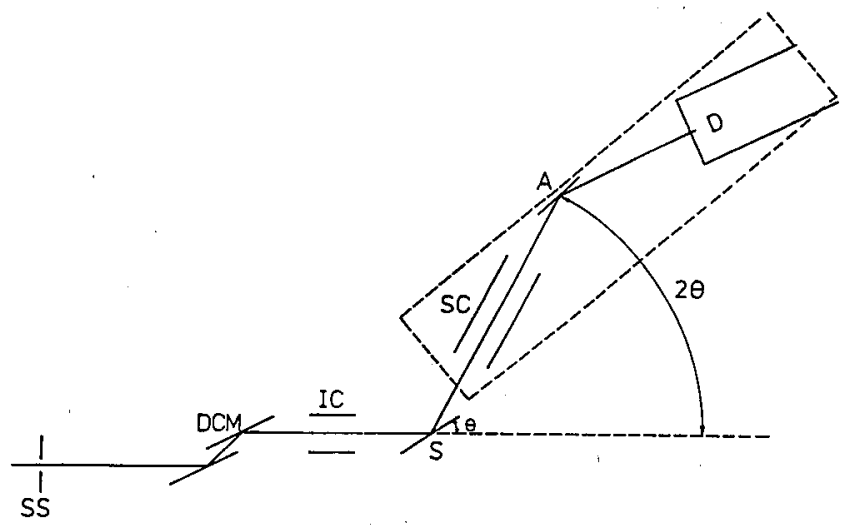

Fig. 1. Beam path: $\mathrm{SS}=$ slit system, $\mathrm{DCM}=$ double crystal monochromator, $\mathrm{IC}=$ ionization chamber (monitor), $\mathrm{S}=$ sample, $\mathrm{SC}=$ Soller collimator (foils parallel to scattering plane), $\mathrm{A}=$ analyser crystal, $\mathrm{D}=$ detector.
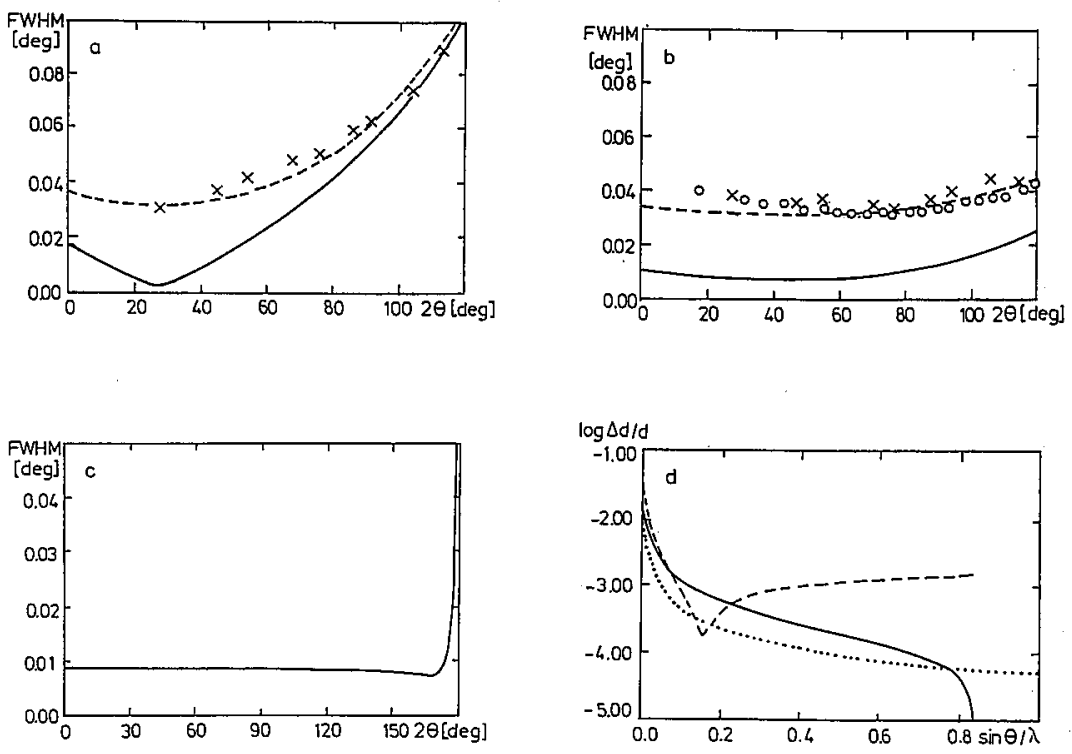

Fig. 2. Resolution functions: (a) Si 111 monochromator and analyser crystals. The full line gives the theoretical resolution. Crosses are measured values. The dashed line is obtained if a broadening of $0.03^{\circ}$ due to sample effects is added to the theory $(\lambda=0.154 \mathrm{~nm})$. (b) Be 004 monochromator and $\mathrm{Si} 111$ analyser. Else as in (a). The circles were obtained with a wavelength of $0.103 \mathrm{~nm}$ from the $\mathrm{Be} 006$ reflection excited simultaneously. (c) Theoretical resolution for a Be 006 monochromator at $\Theta=89^{\circ}$ $(\lambda=0.12 \mathrm{~nm})$. (d) Theoretical resolution for a Be 006 monochromator (full line) and a Si 111 monochromator (dashed line) at the same wavelength of $0.12 \mathrm{~nm}$. The dotted line corresponds to the resolution limited by particle-size broadening of $1 \mu \mathrm{m}$ particles. 
resolution is achieved at high monochromator angles. Using high-order reflections from perfect crystals increases the resolution but entails a dramatic decrease in intensity due to decreasing Darwin widths.

Mosaic crystals can circumvent this problem. They have to be used in a double crystal arrangement to keep the term proportional to the mosaic spread small and must therefore have low absorption to increase the probability of having pairwise parallel blocks in both crystals within the absorption length. Beryllium crystals having a mosaic spread of about one minute of arc have been successfully applied [2]. Figure $2 \mathrm{~b}$ shows the resolution function for such a monochromator angle of $60^{\circ}$. A monochromator operating close to backscattering has been built [3], and first tests gave intensities comparable to those from perfect Si 111 crystals. Due to instabilities caused by thermal effects no experimental resolution function has been determined so far. The theoretical performance of the backscattering monochromator is shown in Fig. 2c and it is compared to that of a Si 111 monochromator at the same wavelength in Fig. 2d. A comparison with the particle-size limit for a grain size of $1 \mu \mathrm{m}$ shows that no further improvement is required.

\section{Peak shape}

The Gaussian approximation has the advantage that it gives an analytical expression for the half widths, but it cannot reproduce the peak shape. For this purpose the convolution integral has to be solved numerically. To obtain the instrumental function an ideal sample is assumed which means that it has infinite mosaic spread and vanishing Darwin width. For the different arrangements of the optical components the expressions for the convolution integrals are given in [1]. Here, without loss in generality, only the triple-axis geometry with perfect crystal monochromator and analyser will be discussed. The integral is then given by

$$
\begin{aligned}
& I(\gamma) \approx \iint \mathrm{d} \lambda \mathrm{d} \alpha F(\alpha) D_{1}\left(\alpha-\Delta \lambda / \lambda \tan \Theta_{1}\right) \\
& \times D_{3}\left(\gamma+\alpha+\Delta \lambda / \lambda\left(2 \tan \Theta_{2}-\tan \Theta_{3}\right)\right)
\end{aligned}
$$

where $D_{1,3}$ are the Darwin curves of monochromator and analyser and $F$ describes the angular distribution of the incoming radiation. The angular parameters $\alpha$ and $\gamma$ describe the direction of the incoming and scattered radiation, while $\Delta \lambda / \lambda$ describes the wavelength spread.

Each factor in the integral can in principle be calculated or measured. In practice however, the angular distribution of the incoming radiation is not easy to calculate, because it depends on various parameters of the source and the beamline which furthermore may change with the operating conditions of the storage ring. Detailed investigations have been performed for the case that a focusing mirror is used in front of the monochromator. A toroidal mirror changes the angular distribution of the beam [4], since due to its meridional curvature parallel rays travelling at different heights will be reflected under different angles, and due to the sagittal curvature rays travelling at the same height are reflected under different angles, because the outer rays hit the mirror before the central rays. These two effects convert a parallel bundle into a rather asymmetrical distribution 
which is further modified due to cut-off effects at the ends of the mirror. Figure 3a shows measured peak shapes as function of the vertical position of the mirror, and Fig. $3 \mathrm{~b}$ shows the corresponding peak shapes obtained theoretically by ray tracing. For the ray-tracing calculations a well-aligned beamline was assumed. Differences between theory and experiment might be due to misalignments.

Another approach is based on the profile that is measured by scanning the analyser crystal through the monochromatic beam. For this profile the theoretical curve is given by

$$
I(\gamma) \approx \iint \mathrm{d} \lambda \mathrm{d} \alpha F(\alpha) D_{1}\left(\alpha-\Delta \lambda / \lambda \tan \Theta_{1}\right) D_{3}\left(\gamma+\alpha+\Delta \lambda / \lambda \tan \Theta_{3}\right)
$$

Comparison with Eq. (2.1) shows that only the argument of the last term is different. Deconvolution of this analyser curve from the measured profile (2.2) followed by a convolution with a scaled analyser curve gives the instrumental function. A first approach in which the widths of the Darwin curves were neglected and replaced by $\delta$-functions is shown in Fig. 3c.

\section{Investigation of moving objects}

The above calculations are based on the assumption that misalignments or effects such as sample geometry and finite penetration depth do not affect the peak shape. This is indeed the case if an angular sensitive device like a set of parallel foils or an analyser crystal is installed in front of the detector (Figs. 4a, b). This low sensitivity to sample geometry and position can be applied in the investigation of moving objects. A demonstration experiment was performed on the rotor blades of a turbo pump [5]. Figure $4 \mathrm{c}$ shows the setup of the experiment while Fig. $4 \mathrm{~d}$ shows the $\mathrm{Al}(333),(511)$ reflection from the rotor blades once at rest and once rotating at $50000 \mathrm{rpm}$. The peak shift towards higher angles is due to the contraction of the lattice planes perpendicular to the action of the centrifugal force.

\section{Diffraction under grazing incidence}

Another application of this geometry is the investigation of thin layers under grazing incidence. The effective penetration depth $t_{\text {eff }}$ decreases with decreasing angle of incidence $\alpha\left(t_{\mathrm{eff}}=t_{\mathrm{abs}} \sin \alpha, t_{\mathrm{abs}}=\right.$ absorption length). The width of the diffracted beam increases. But with the use of an analyser crystal or a set of parallel foils in front of the detector the peak will neither be broadened nor will its position be affected by geometrical effects. If $\alpha$ approaches the critical angle of total reflection $\alpha_{\mathfrak{c}}$, there is however a peak shift due to refraction.Figures $5 \mathrm{a}$ and $5 \mathrm{~b}$ show the 111 reflections from a sample consisting of 3 layers $(100 \mathrm{~nm}$ each) of $\mathrm{Al}$ on $\mathrm{Ag}$ on $\mathrm{Au}$ on a glass carrier. These elements all have a fcc structure and similar lattice constants $\left(a_{\mathrm{Al}}=0.405 \mathrm{~nm}, a_{\mathrm{Ag}}=0.409 \mathrm{~nm}, a_{\mathrm{Au}}=0.408 \mathrm{~nm}\right)$. Furthermore, they exhibit relatively broad peaks which therefore overlap as can be seen in Fig. 5a showing a $\Theta-2 \Theta$-scan. If the pattern is taken at a constant angle of incidence $\left(\alpha=0.305^{\circ}\right)$, it looks completely different. The Au peak has vanished due to the decrease in $t_{\text {eff }}$ which is in this case not only due to geometrical effects, but also because $\alpha$ is below $a_{\mathrm{c}}$ of $\mathrm{Ag}$. This lower penetration depth in the $\mathrm{Ag}$ 

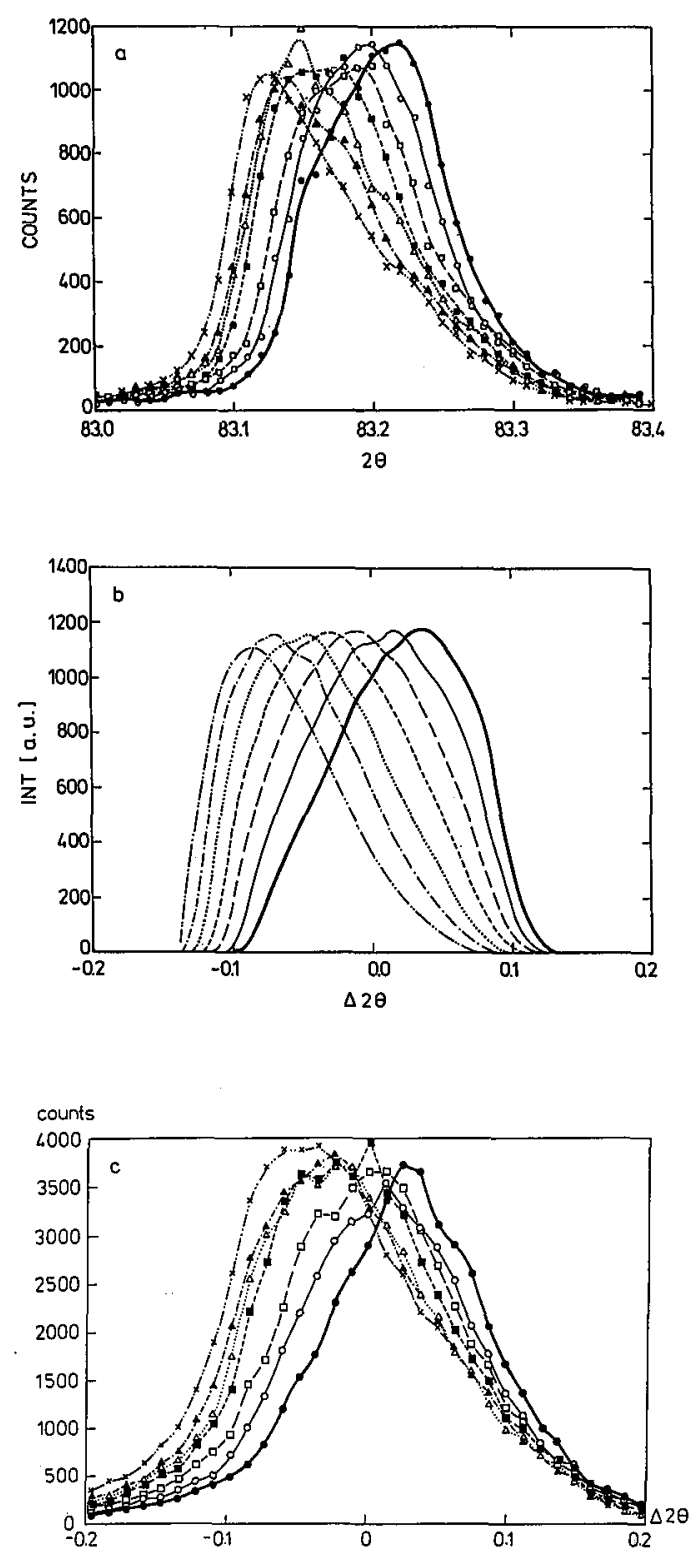

Fig. 3. Peak shapes: (a) 420 reflection of $\mathrm{LaB}_{6}, \lambda=0.13 \mathrm{~nm}$. Between the different scans the mirror was moved downward in steps of $0.5 \mathrm{~mm}$ (in the order: full fat, full, long dashed, short dashed, dotted, dash dotted, dash double dotted). (b) Ray-tracing calculations using the same parameters as for the experiment. (c) Profiles obtained by scanning the analyser through the primary beam and scaling the angular scale with the factor $1-2 \tan \Theta_{2} /\left(\tan \Theta_{1}+\tan \Theta_{3}\right)$. 

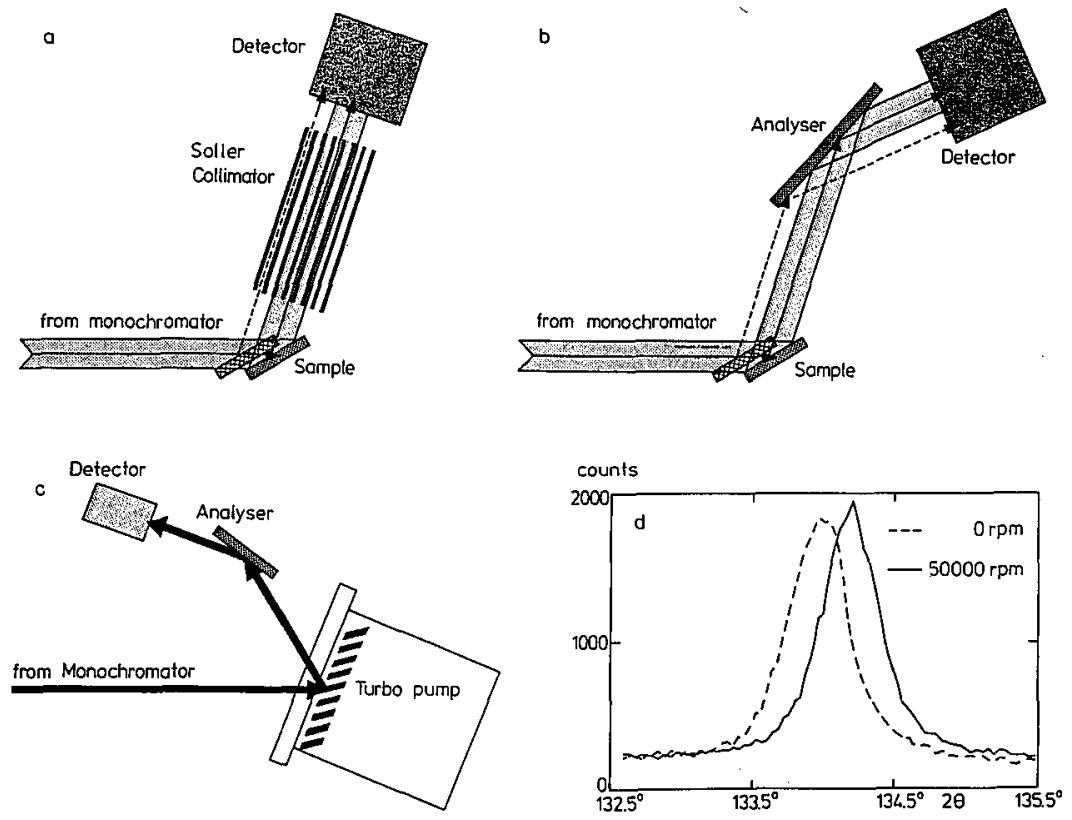

Fig. 4. Investigation of moving objects: (a) and (b) demonstrate that the setup is aberration-free, (c) shows the measuring scheme and (d) the peak shift due to induced strain (dashed line: rotor blades at rest, solid line: rotating at $50000 \mathrm{rpm}$ ).
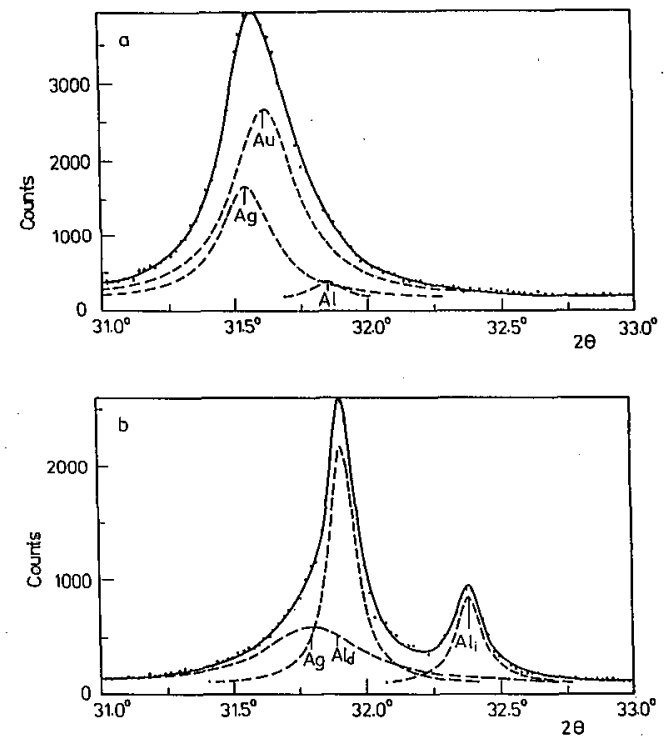

Fig. 5. Diffraction under grazing incidence on a sample consisting of thin layers of $\mathrm{Al}$ on $\mathrm{Ag}$ on $\mathrm{Au}$ : (a) was taken in a $\Theta-2 \Theta$-scan, (b) at a constant angle of incidence of $0.305^{\circ}$. 


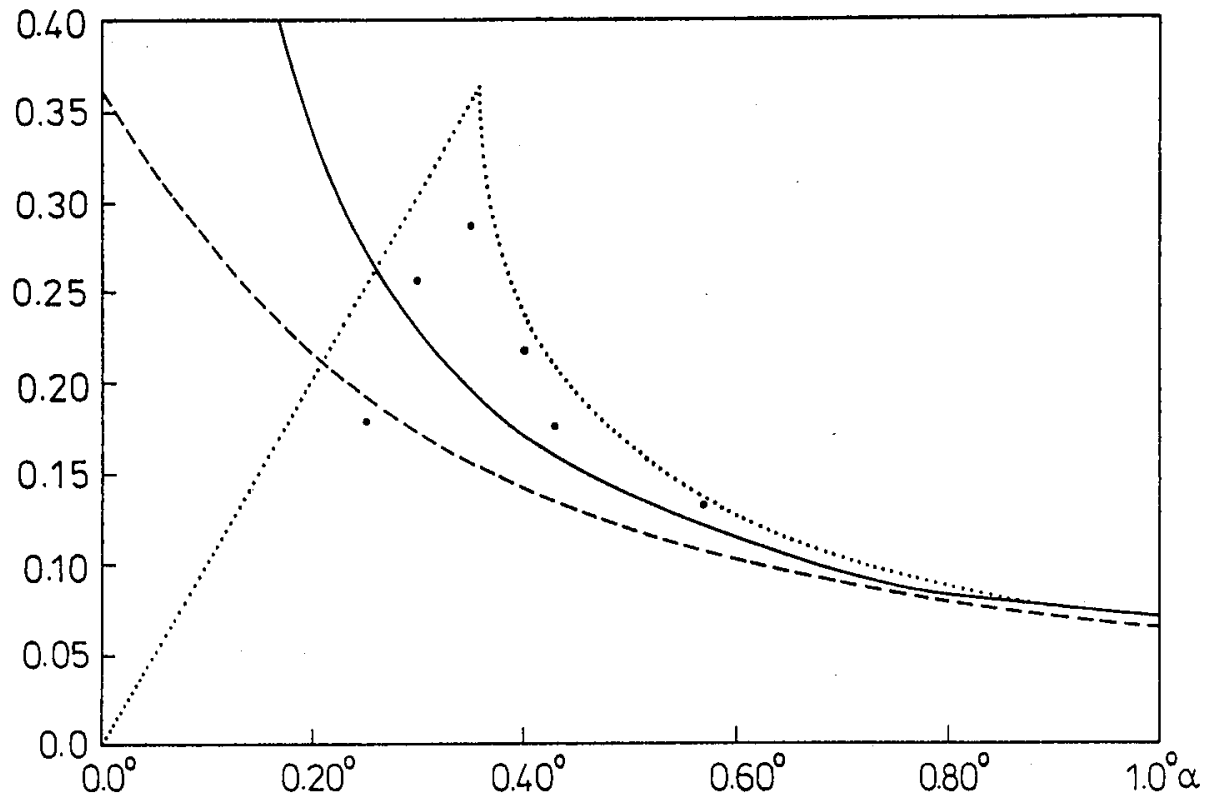

Fig. 6. Diffraction correction for three different models. Full line - dynamical theory, dashed line - extended dynamical theory, dotted line - geometrical theory plus Snell's law, crosses - experiment.

layer also gives rise to particle-size broadening of the $\mathrm{Ag}$ peak. Furthermore, an additional peak $\mathrm{Al}_{\mathrm{i}}$ appears due to diffraction of the total reflected beam. Both the $\mathrm{Ag}$ and the $\mathrm{Al}$ peak have shifted towards higher angles due to refraction. The peak positions including refraction effects are given by the dynamical theory of $\mathrm{X}$-ray diffraction. If $\alpha$ approaches $\alpha_{\mathrm{c}}$, the approximation that the tangentials to the dispersion surface are planes will lead to an infinite peak shift.

An extended dynamical theory [6] that uses spheres as tangentials gives a finite peak shift that approaches $\alpha_{\mathrm{c}}$, if $\alpha$ approaches 0 . Another approach simply uses the geometrical theory and accounts for diffraction by applying Snell's law. It gives a maximum displacement of $\alpha_{\mathrm{c}}$ for $\alpha=\alpha_{\mathrm{c}}$ and zero displacement for $\alpha=0$. The data obtained from the above-mentioned experiment tend to support the last approach (Fig. 6). There is, however, some uncertainty because of the severe overlap of the three reflections. An experiment on a simple system will allow a final decision.

The exact knowledge of the refraction correction is of importance in the determination of residual strain by the $\sin ^{2} \psi$ method. If it is not considered, the peak shift towards higher angles simulates the effects of high compressive strain. One may circumvent this problem by performing the experiment not in the $\omega$-mode, but in the $\psi$-mode. 


\section{The translation powder diffractometer at HASYLAB}

A conventional two-circle diffractometer is not sufficient to allow $\omega$ or $\psi$ scans, and further experiments such as the measurement of pole figures or the investigation of structural transitions, require specialised setups such as an Eulerian cradle, cryostats, furnaces etc. To achieve high flexibility fast exchange of such components is required. Furthermore, external groups must have the ability to install equipment from their home laboratories without major modifications. The translation diffractometer realised at HASYLAB beamline B2 fulfils these requirements [7]. It consists essentially of two circles facing each other which can be moved along their common axis (Fig. 7). On either circle self centering base plates can be mounted. Arbitrary instrumentation can thus be installed and preadjusted

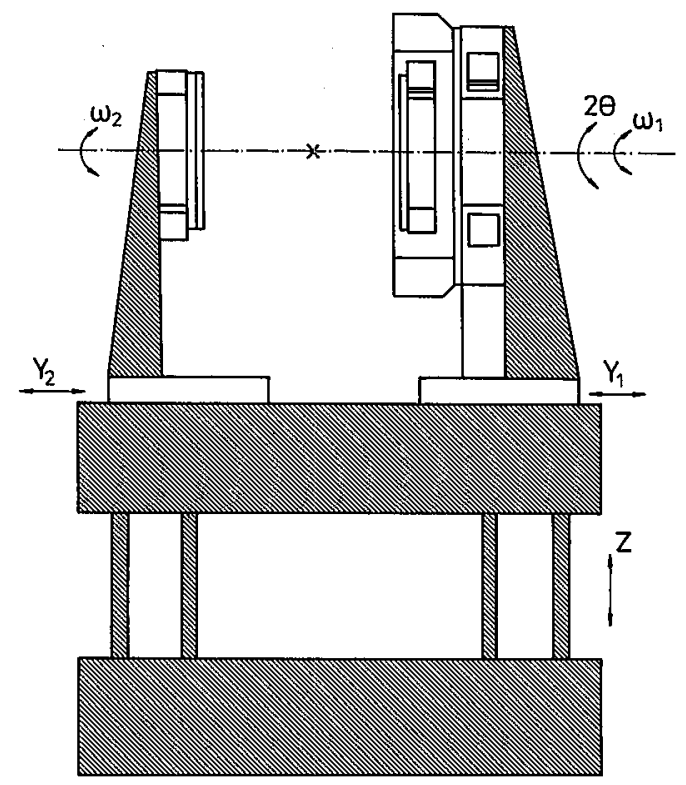

Fig. 7. The translation diffractometer.

on these base plates and then fixed to one of the circles which will then bring the attachment into the beam.

For the $\omega$-circle, holders for capillaries and flat specimens, as well as a cryostat $(12 \mathrm{~K})$ and a furnace $\left(1000^{\circ} \mathrm{C}\right)$ are available. Attachments for the $2 \Theta$-circle are a two-circle goniometer for analyser crystal and detector on which alternatively a Soller collimator can be mounted or a diode array as position-sensitive detector. The Eulerian cradle for the circle carries an $X Y Z$-table on which different holders can be mounted. Some holders allow the application of a load on the sample. This configuration allows the investigation of the strain distribution within single grains in a polycrystalline matrix (Fig. 8) [8]. 

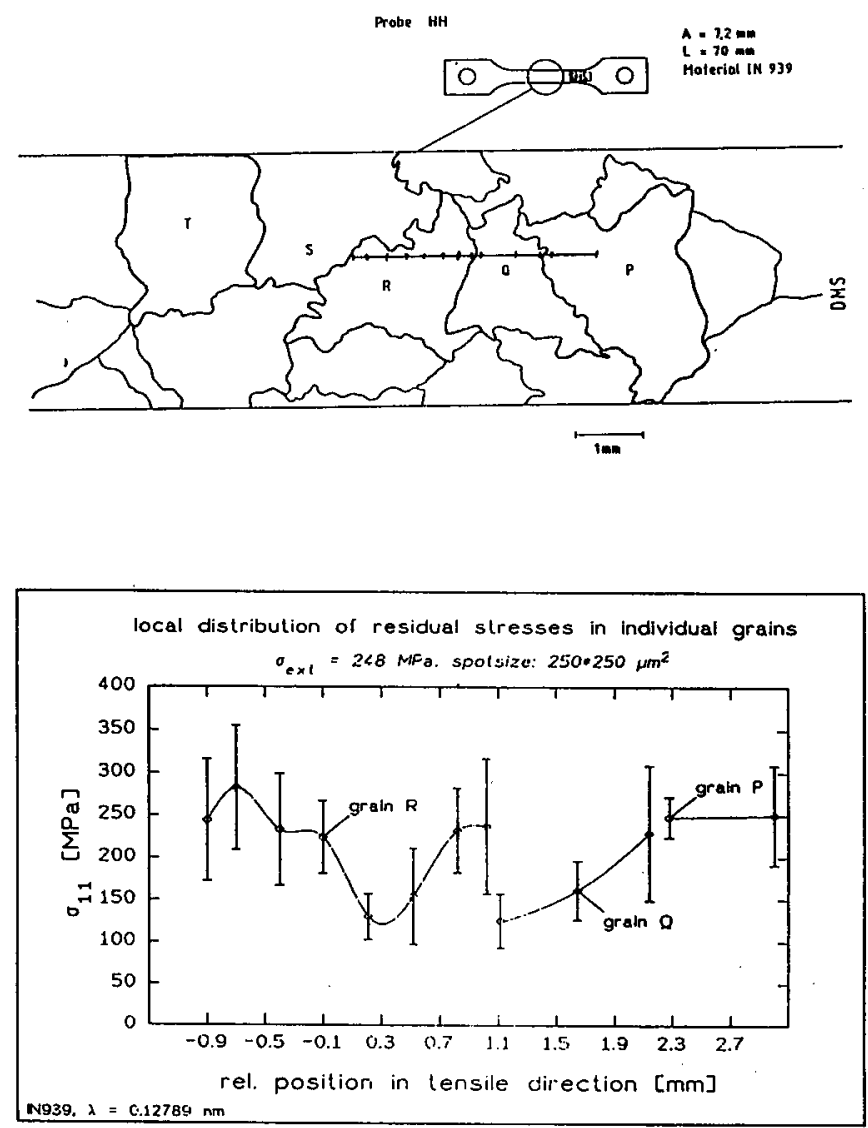

Fig. 8. Strain as function of position in three grains in a polycrystalline matrix.

\section{Time-resolved experiments}

The position-sensitive detector is especially suited for time-resolved measurements because step scanning is too time consuming. If however a larger part of the pattern is investigated, the integral count rate increases and the dead time of the detector becomes a problem. Therefore, integrating devices like film, image plates, or diode arrays are used in preference to single photon counting devices. An electronic detector like the diode array allows for on-line observation. The area of such a device is however rather small ( 1 inch) so that only a small region can be observed. A decrease in the sample-to-detector distance extends the angular range, but entails a lower resolution. For the patterns in Fig. 10 the sample-to-detector distance was $50 \mathrm{~mm}$. With a sample diameter of $0.3 \mathrm{~mm}$ this gives a resolution of $6 \mathrm{mrad}=0.35^{\circ}$ which is still sufficient for many applications. The upper two diagrams of Fig. 10 show patterns of $\mathrm{CaF}_{2}$ taken with $20 \mathrm{~s}$ (Fig. 9a) and $0.2 \mathrm{~s}$ (Fig. 9b) exposure time. If, rather than perfect crystals, a synthetic multilayer delivering 

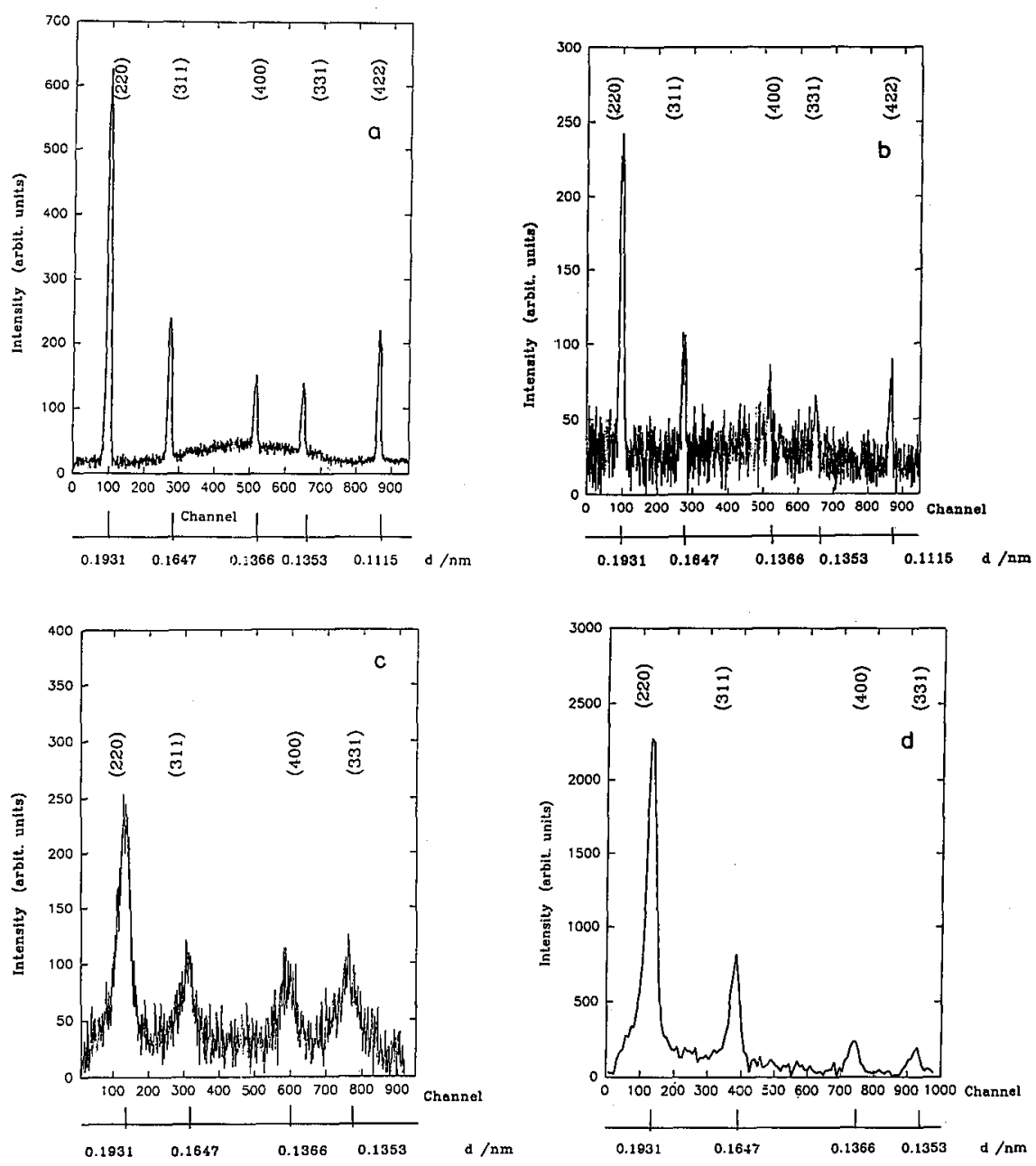

Fig. 9. Pattern of $\mathrm{CaF}_{2}$ taken with difterent exposure times. (a) Si 111 monochromator, $20 \mathrm{~s}$, (b) Si 111 monochromator, $0.2 \mathrm{~s}$, (c) multilayer, $16 \mathrm{~ms}$, (d) multilayer, $2.5 \mathrm{~ms}, 20$ diodes form one detector element.

a broader energy band is used, the time resolution can be further increased [9]. The pattern in Fig. 9c was taken within 16 milliseconds, and here the time resolution was limited by the read-out time for the 1024 single photodiodes. If they are read out in groups of 20, this time reduces to 2.5 milliseconds (Fig. 9d).

One application for such a high time resolution is the investigation of solid flames [10]. These reaction can neither be slowed down nor investigated by stroboscopic methods. Figure 10 shows the reaction of $\mathrm{Ta}$ with $\mathrm{C}$ to form $\mathrm{TaC}$ in which first the intermediate $\mathrm{Ta}_{2} \mathrm{C}$ is formed. If higher resolution and insensitivity to the sample geometry is required, a continuous scanning method can be applied [11]. Figure 11 shows a series of patterns taken during the crystallisation of $\mathrm{C}_{20} \mathrm{H}_{42}$ from 


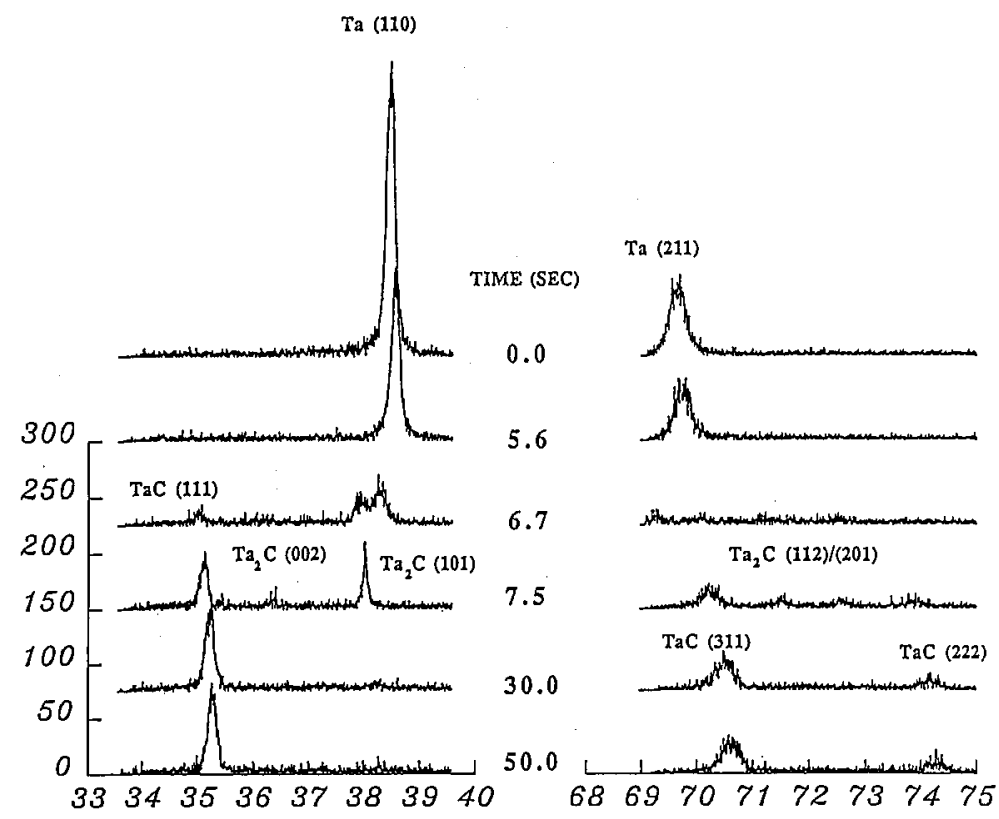

$2 \theta$

Fig. 10. Reaction of $\mathrm{Ta}$ and $\mathrm{C}$ to $\mathrm{TaC}$. It can be seen that the intermediate $\mathrm{Ta}_{2} \mathrm{C}$ is formed.

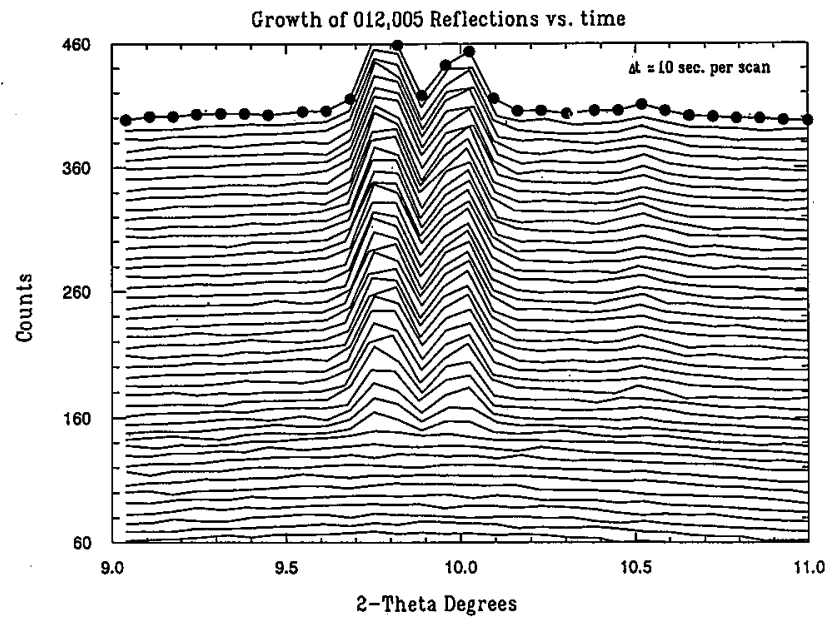

Fig. 11. Crystallisation of hydrocarbons. Two reflections from $\mathrm{C}_{20} \mathrm{H}_{42}$ develop while the contribution of the liquid to the total scattering decreases. 
solution in $\mathrm{C}_{12} \mathrm{H}_{26}$. They were taken with a Si 220 monochromator and a Soller collimator in front of the detector. The angular resolution is about 0.1 degree. A position-sensitive detector could not be used because the rather large scattering volume required would have produced broad peaks.

\section{Anomalous dispersion experiments}

The white spectrum of synchrotron radiation allows free choice of the wavelength. This can be applied in anomalous dispersion experiments. Several single crystal diffraction methods ( $\lambda$-method, $\Delta$-synthesis, FRED, etc.) have been developed [12] which can be partially applied to powder diffraction. Recently a novel method has been introduced which allows the phase determination from powder data $[13,14]$.

While in single crystal diffraction the reflections of a Bijovet pair are observed separately they coincide in powder diffraction because their lattice vectors only differ in sign. The intensities of the two reflections vary with the contribution of anomalous scattering. The structure factor is given by

$$
F(h)=\sum_{i} f_{i} \mathrm{e}^{2 \pi \mathrm{i} h r_{i}}
$$

where $f_{i}$ is the atomic form factor and $r_{i}$ is the position of the $i$-th atom in the unit cell. $h$ is the reciprocal lattice vector. For the case of one anomalous scatterer with $f=f_{0}+f^{\prime}+\mathrm{i} f^{\prime \prime}$ we get

$$
F(h)=\sum_{i} f_{0 i} \mathrm{e}^{2 \pi \mathrm{i} h r_{i}}+\left(f^{\prime}+\mathrm{i} f^{\prime \prime}\right) \mathrm{e}^{2 \pi \mathrm{i} h r_{n}}=\left|F_{0}\right| \mathrm{e}^{\mathrm{i} \Phi}+\left(f^{\prime}+\mathrm{i} f^{\prime \prime}\right) \mathrm{e}^{\mathrm{i} \psi}
$$

and analogously

$$
F(-h)=\left|F_{0}\right| \mathrm{e}^{-\mathrm{i} \Phi}+\left(f^{\prime}+\mathrm{i} f^{\prime \prime}\right) \mathrm{e}^{-\mathrm{i} \psi} \text {. }
$$

For centrosymmetrical structures $\Phi$ and $\psi$ can only have the values 0 or $\pi$, and assuming that the anomalous scatterer is located at the inversion center we obtain

$$
F(h)= \pm\left|F_{0}\right|+\left(f^{\prime}+f^{\prime \prime}\right)=F(-h) \text {. }
$$

The amplitudes and thus the intensity of both reflections suffer the same change compared to a reference measurement. The direction depends on whether $\Phi$ is 0 or $\pi$ (Fig. 12). Figure 13 shows two patterns from $\mathrm{CuSO}_{4}$. One was taken with

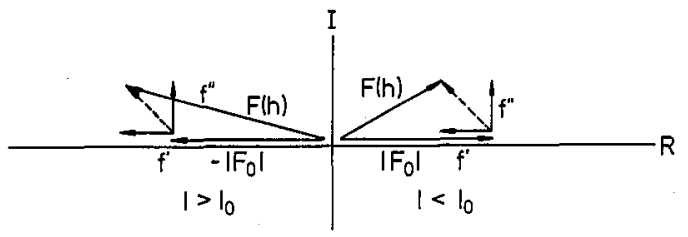

Fig. 12. Change of the scattering amplitude due to an anomalous scatterer.

a wavelength close to the $\mathrm{Cu} K$-edge, the other with half this wavelength. The change of the relative intensities is evident. For the case of non-centrosymmetrical structures two different anomalous scatterers are needed and three measurements at different wavelengths must be performed. This general case is treated in [12]. 


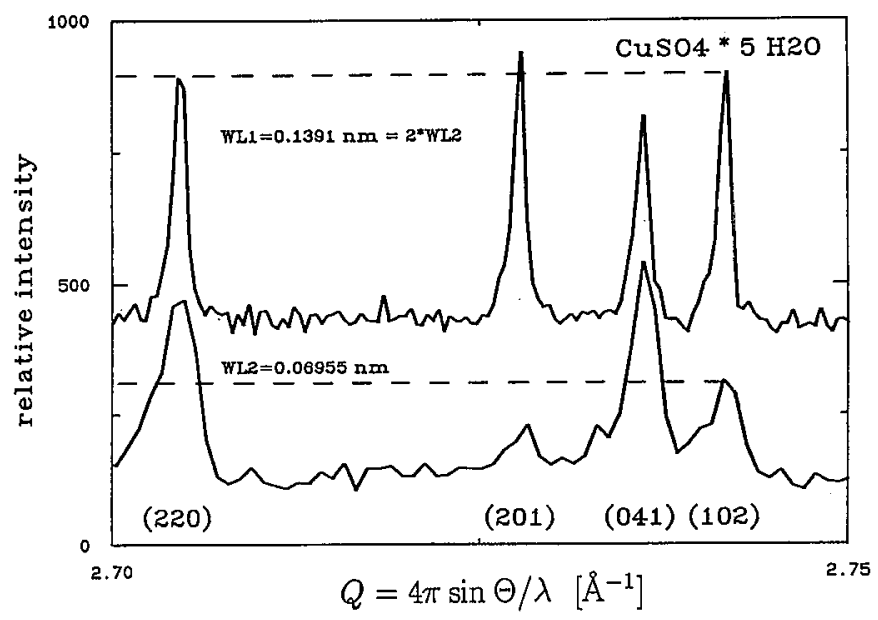

Fig. 13. Pattern of $\mathrm{CuSO}_{4}$ taken once close to the $\mathrm{Cu} K$-edge and once at half the wavelength.

\section{Summary}

Powder diffraction with synchrotron radiation covers a wide field of applications. In crystallography not only accurate structure analysis and refinement due to the high resolution but also phase determination is possible. Material sciences profit from the narrow peaks of known shape as well as from the aberration-free setup or the high intensity of the radiation. Chemical applications are no longer restricted to structure analysis but also kinetic studies on the millisecond time scale have become possible. The intention of this article was to show these new possibilities. Information about the numerous experiments which have become routine can be found in the annual reports of the synchrotron radiation laboratories or in an increasing number of publications. Synchrotron radiation has led to a revolution in powder diffraction by introducing many new methods but there still remains a lot to discover in this exciting field.

\section{References}

[1] T. Wroblewski, Acta Crystallogr. A 47, 571 (1991).

[2] T. Wroblewski, in: 2nd European Conf. Prog. Synchrotron Radiat. Res., Conf. Proc., Vol. 25, Eds. A. Balerna, E. Berniere, S. Mobilio, SIF, Bologna 1990, p. 319.

[3] T. Wroblewski, W. Ternes, Rev. Sci. Instrum. 63, 1091 (1992).

[4] S.M. Heald, J.B. Hastings, Nucl. Instrum. Meth. 187, 533 (1981).

[5] J. Ihringer, A. Küster, J.K. Maichle, T. Wroblewski, J. Appl. Crystallogr. 21, 972 (1988).

[6] F. Rustichelli, Philos. Mag. 31, 1 (1975).

[7] H. Arnold, H. Bartl, H. Fuess, J. Ihringer, K. Kosten, U. Löchner, P.U. Pennartz, W. Prandl, T. Wroblewski, Rev. Sci. Instrum. 60, 2380 (1989). 
[8] H.-A. Crostack, M. Wrobel, U. Selvadurai-Laßl, Biese, T. Wroblewski, in: HASYLAB-Jahresbericht 1991, HASYLAB, Hamburg 1991, p. 295.

[9] P.U. Pennartz, U. Löchner, H. Fuess, T. Wroblewski, to be published in J. Appl. Crystallogr.

[10] J. Wong, E.M. Larson, J.B. Holt, P.A. Waide, B. Rupp, R. Frahm, Science 249, 1406 (1990).

[11] S. Doyle, in: HASYLAB-Jahresbericht 1991, HASYLAB, Hamburg 1991, p. 299.

[12] K. Eichhorn, Acta Phys. Pol. A 82, 51 (1992).

[13] W. Prandl, Acta Crystallogr. A 46, 988 (1990).

[14] W. Limper, W. Prandl, T. Wroblewski, Mater. Sci. Forum 79-82, 221 (1991). 